\title{
Planification des infirmiers anesthésistes : analyse comparative des performances de différents solveurs
}

\author{
Lorraine Trilling $^{[1][2]}$, Alain Guinet ${ }^{[1]}$, Dominique Le Magny ${ }^{[3]}$
}

[1] INSA de Lyon, Laboratoire PRISMa, 20 av. Albert Einstein, 69100 Villeurbanne Email. : lorraine.trilling@insa-lyon.fr

[2] Université de Saint-Etienne, Laboratoire LASPI, 12 av. de Paris, 42300 Roanne

[3] Service d'Information Médicale, Centre Hospitalier de Valence, 26000 Valence, France

Résumé. L'affectation des infirmiers aux différentes vacations de soins est une activité réputée difficile et souvent réalisée à la main. Dans cet article nous nous penchons sur le problème de la planification des infirmiers anesthésistes du bloc opératoire, qui consiste à affecter à chaque infirmier pour chaque jour de la semaine, une vacation correspondant à une journée (ou une nuit) de travail. Des activités de surveillance de salle de réveil, de prise en charge du patient en chirurgie réglée ainsi qu'en chirurgie d'urgence doivent être couvertes et des contraintes respectées, tout en recherchant un objectif d'équité de répartition des vacations entre les infirmiers. Plusieurs solveurs libres et commerciaux sont comparés dans le cadre de la résolution de ce problème.

Mots clés. Planification de personnel, planning non cyclique, programmation linéaire, solveur, transférabilité, bloc opératoire.

\section{Introduction}

Dans un contexte budgétaire restrictif, obligeant les hôpitaux à travailler avec des enveloppes limitées, la rationalisation des coûts s'impose. L'une des stratégies préconisées est le regroupement de moyens qui se matérialise dans le secteur chirurgical par la création de blocs 
opératoires pluridisciplinaires de grandes tailles, appelés également plateaux médicotechniques (PMT). Cette mutualisation des ressources pose de nombreux problèmes organisationnels et opérationnels, tant au niveau humain que matériel. La répartition adéquate du personnel infirmier et soignant est donc une préoccupation majeure des directions de département infirmier et des cadres de santé. En effet le personnel représente plus de $75 \%$ du budget de l'hôpital (Chan, 2002), et son emploi doit être optimisé afin de réduire les coûts de fonctionnement.

Le bloc opératoire, que l'on appellera par la suite plateau médico-technique (PMT), est une infrastructure qui comporte deux secteurs: les salles d'intervention et les salles de surveillance post-interventionnelles (SSPI). Au sein du PMT travaillent différentes catégories professionnelles, du domaine médical, soignant, administratif et de maintenance. Chacune de ces catégories doit être gérée de manière à garantir un bon fonctionnement du PMT mais aussi la satisfaction du personnel, qui est un élément non négligeable impactant la qualité des soins délivrés aux patients. L'affectation de vacations journalières à des infirmiers sur une période de plusieurs jours voire plusieurs semaines est une tâche difficile et coûteuse en temps, bien souvent encore réalisée à la main par les cadres de santé. Il existe des logiciels de gestion de temps incluant des fonctions de planification (GMSIH, 2002), mais elles sont souvent peu utilisées car ne prennent pas en considération les spécificités de chaque problème de planification.

Notre ambition est de proposer aux cadres de santé responsables de l'élaboration des plannings infirmiers, un outil informatique permettant de faciliter l'affectation des différentes vacations aux employés sur un horizon de planification. Notre effort de recherche s'est concentré sur la planification des infirmiers anesthésistes diplômés d'Etat (IADE), qui en raison de leur activité transversale sur un grand nombre de spécialités (i.e., chirurgie viscérale, orthopédie, ophtalmologie, etc.) et sur plusieurs tâches (i.e., prise en charge de l'anesthésie, 
surveillance du réveil, etc.), sont organisées en pool de personnels le plus souvent polyvalent en anesthésie. Cette polyvalence des personnels accroît le nombre de possibilités d'affectation aux activités, et laisse ainsi plus de marge de manœuvre dans la réalisation des plannings. La polyvalence des personnels constitue néanmoins une contrainte additionnelle à prendre en compte : le maintien des compétences dans chaque spécialité et sur chaque type d'activité nécessaire à la polyvalence passe inévitablement par une pratique régulière. Celle-ci est rendue possible si une attention particulière est portée à l'harmonisation de la répartition des tâches entre les personnels et à leur affectation régulière sur les différentes spécialités et les différentes tâches. Le modèle que nous proposons tient compte de cette homogénéité nécessaire dans la répartition des tâches, non seulement dans les contraintes à considérer mais aussi dans l'objectif à optimiser.

Les activités des IADE se concentrent principalement sur les heures d'ouverture du bloc opératoire (généralement de 8 h00 à $16 \mathrm{~h} 00$ les jours de semaine), mais l'obligation de permanence des soins contraint les établissements à mettre en place des gardes pour les soirs, les week-end et les jours fériés. Ainsi des vacations permettant d'assurer la prise en charge des cas urgents se présentant doivent être couvertes (en général un à deux infirmiers par vacations). De par cette spécificité et la variété des activités prises en charge par les IADE, le problème de planification des infirmiers anesthésistes (PPIA) se différentie du problème « classique » de planification des infirmiers d'unité de soins (communément désignée dans la littérature comme le «Nurse Scheduling Problem» ou le «Nurse Rostering Problem» (Burke et al., 2004)), pour lequel des soins continus doivent être assurés (24h sur 24, 7 jours sur 7) et sont répartis selon trois types de vacations (Matin/Après-midi/Nuit).

Dans cet article nous proposons une modélisation du problème de planification des infirmiers anesthésistes (PPIA) sous forme d'un programme linéaire à variables mixtes, dont l'objectif recherché est la maximisation de l'équité de la répartition des tâches entre les personnels. 
Notre choix s'est porté sur la programmation linéaire qui a montré son efficacité pour la modélisation et la résolution du PPIA, en comparaison à l'utilisation de la programmation par contraintes pour le même problème (Trilling et al., 2006). Nous comparons les résultats obtenus par différents solveurs (commerciaux et libres) pour la résolution de ce problème linéaire mixte, afin de vérifier l'efficacité d'un solveur libre par rapport à des solveurs de référence, et de pouvoir fournir aux cadres de santé des outils utilisables sans avoir à investir spécifiquement dans un logiciel payant.

Dans une première section, cet article s'attache à présenter un bref état de l'art sur les problèmes de planification de personnels. La seconde section est consacrée à la description précise du problème de planification des infirmiers anesthésistes (PPIA), ainsi qu'à sa modélisation sous la forme d'un programme linéaire en nombres mixtes. Dans une troisième section nous comparons les comportements de différents solveurs du marché (commerciaux et libres) lors de la résolution d'un large panel de problèmes de différentes tailles (effectif, salles opératoires, horizon temporel). Finalement nous concluons et donnons quelques perspectives de recherche.

\section{Etat de l'art}

La planification du personnel (Staff scheduling), correspond à la construction d'emplois du temps spécifiant pour chaque employé ses jours et horaires de travail, ainsi que ses jours de repos, tout en respectant un ensemble de contraintes. Celles-ci sont multiples (législation du travail, conventions collectives, couverture de la charge, préférences individuelles, etc.) et dépendent du domaine d'application (Weil et al., 1995; Ernst et al., 2004). Elles peuvent être considérées comme fermes (obligatoires) ou souples (à respecter au mieux). Lors de la construction d'un programme de travail, le but est de trouver le meilleur arbitrage possible 
entre trois critères : le coût, la qualité de service et la satisfaction sociale, comme indiqué sur la figure 1 (Partouche, 1998). Par qualité de service on entend la réalisation des tâches de production de biens ou de services dans les meilleurs délais. Dans le secteur des services comme les organisations de production de soins, où le travail ne peut pas être stocké, la couverture de la charge doit permettre de répondre à un besoin avec un personnel en nombre suffisant au bon moment. Le critère de coût recouvre les frais engendrés par les salaires, les formations, mais aussi les coûts liés aux éventuels dysfonctionnements engendrant par exemple des heures supplémentaires. Le critère de satisfaction sociale se mesure par le degré de satisfaction des préférences communes (maximiser l'équité et l'homogénéité dans les plannings, privilégier les jours de repos consécutifs) et des préférences individuelles des employés (respect des desideratas au niveau des jours de congés et des horaires de travail). La fonction objectif peut alors être construite pour minimiser le coût (salaires, heures supplémentaires, etc.), maximiser la qualité du service (couverture de la charge) ou bien maximiser la satisfaction des employés (minimisation des contraintes souples violées, maximisation de l'équité entre les individus) (Blöchliger, 2004).

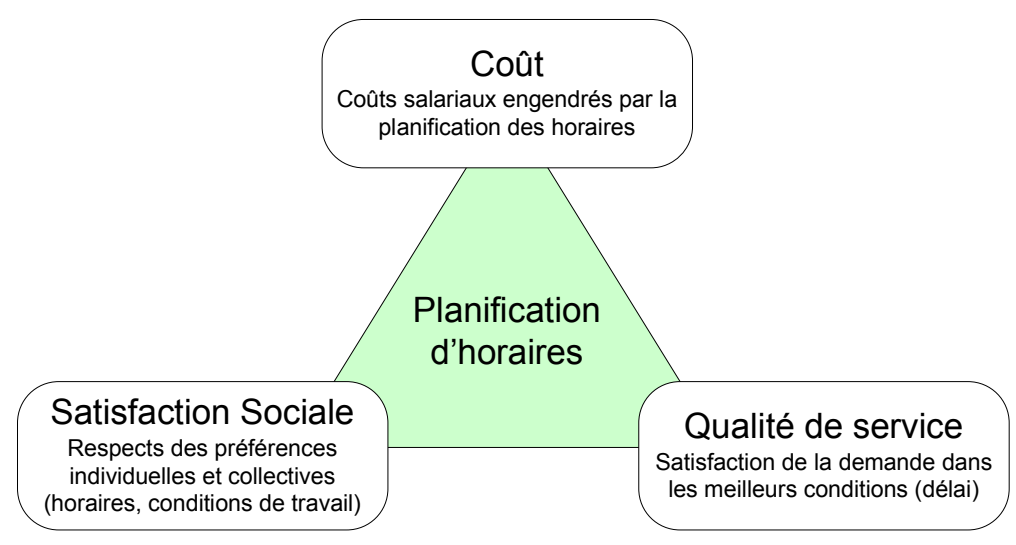

Figure 1. Les critères de mesure d'un bon planning

Dans la littérature, plusieurs types de planification sont présentés : les plannings cycliques et non cycliques. Une approche des plus courante est de construire des plannings cycliques, dans 
lesquels une trame est répétée tant que les contraintes restent inchangées sur une période définie. Ces plannings présentent une facilité de construction mais une difficulté réside dans la prise en compte des souhaits des personnels. Dans un processus de planification non cyclique, un nouveau planning est généré pour chaque période de planification. Le temps passé à l'élaboration du planning est important, mais il présente l'avantage d'être relativement flexible (Valouxis and Housos, 2000). C'est sur un problème de planification non cyclique que se focalise ici notre attention.

Le problème de planification (Scheduling Problem) se subdivise en trois sous problèmes : planification des vacations (Shift Scheduling), planification des jours de repos (Day-off Scheduling) et affectation des employés aux différentes tâches (Task Assignment ou Scheduling). Dans la classification proposée par Tien et Kamiyama (Tien and Kamiyama, 1982), le problème de planification est précédé de deux étapes, qui traitent de problèmes à plus long terme : la prévision de la demande par période (Demand Modelling), la prévision des effectifs nécessaires par vacation (Staffing Problem). Les problèmes de construction d'emploi du temps en général, et les problèmes de planification des infirmiers en particulier, ont fait l'objet de nombreuses recherches chez les scientifiques depuis une quarantaine d'années (Burke et al., 2004), traitant la plupart du temps de problèmes mettant en jeu différents objectifs et différentes contraintes. Parmi les techniques employées on peut citer : la programmation entière et mixte (Jaumard et al., 1998), la programmation par but (Berrada et al., 1996), la programmation par contraintes (Weil et al., 1995; Abdennadher and Schlenker, 1999) et les méta-heuristiques tels que les algorithmes génétiques (Aickelin and Dowsland, 2004), la méthode tabou (Valouxis and Housos, 2000) ou le recuit simulé (Brusco and Jacobs, 1995).

Parmi les méthodes proposées, la programmation linéaire en nombres mixtes (PLM) a montré son efficacité dans des problèmes d'affectation se rapprochant de celui qui nous intéresse dans 
ce papier. Jaumard et al. (1998) proposent une approche exacte permettant d'aborder un problème réel de panification d'infirmiers d'unité de soins incluant un certain degré de flexibilité, sur un horizon de 6 semaines. Les auteurs présentent un modèle mathématique sous forme de programme linéaire en nombres entiers (variables binaires) composé de deux problèmes hiérarchisés : (1) un problème principal d'allocation des infirmiers, dont l'objectif est de déterminer la configuration des plannings individuels qui minimise le coût des salariés et maximise la satisfaction des infirmiers (respect des préférences personnelles), tout en respectant strictement les contraintes de charge (qualité de service); (2) un problème auxiliaire de détermination des plannings individuels acceptables satisfaisant les contraintes collectives liées à la charge de travail, aux rotations, aux jours de repos. L'aspect coût est modélisé par des variables d'écart représentant la différence entre le niveau de service requis et le nombre d'infirmiers affectés : ces variables correspondent au personnel supplémentaire auquel il faut faire appel pour couvrir la charge. Pour la résolution de ces deux problèmes, les auteurs ont spécifiquement développé des algorithmes qui leur sont propres (algorithme de séparation et évaluation, et algorithme pseudo-polynômial). Dans notre étude, nous avons privilégié l'utilisation de solveurs de programmation linéaire et entière, qui offrent une certaine facilité de modélisation, et sont selon nous plus aisément transférables aux gestionnaires opérationnels que des algorithmes spécifiques. Nous considérons par ailleurs les affectations des infirmiers de manière journalière et non pas globalement sur l'horizon. Ainsi, la construction des plannings acceptable est réalisée dans le même temps que l'affectation. Enfin, l'aspect coût lié au personnel supplémentaire devant compléter l'effectif en place n'est pas considéré. Par ailleurs, nous introduisons un aspect de recherche d'équité lié à la pénibilité du travail qui est absente chez (Jaumard et al., 1998). 


\section{Problème de planification des infirmiers anesthésistes (PPIA)}

\subsection{Description du problème}

Les infirmiers anesthésistes diplômés d'Etat (IADE) sont les proches collaborateurs de médecins anesthésistes réanimateurs (SFAR, 2002). Ils interviennent à différents stades du processus de prise en charge du patient, sur le site d'anesthésie, en salle de surveillance postinterventionnelle (SSPI) et dans les unités d'hospitalisation. Ils assurent diverses fonctions et principalement :

- La prise en charge des malades en salle, la préparation et le contrôle de l'anesthésie,

- La supervision de la SSPI.

Dans les établissements ayant une fonction d'accueil des urgences chirurgicales, une continuité du service doit être assurée pour l'activité de préparation et de contrôle de l'anesthésie. Les postes correspondent donc à des horaires différents (travail de jour, travail de nuit). L'affectation aux postes peut être journalière, hebdomadaire, mensuelle, annuelle, voir pluriannuelle, cela dépend du degré de polyvalence des infirmiers, du niveau de mutualisation des ressources et de la politique d'affectation choisie par l'hôpital, les cadres de santé et les employés.

Le problème présenté dans ce papier est issu d'une situation de terrain observée dans un hôpital français, dont le plateau médico-technique est composé de 9 salles d'intervention. Il pourrait cependant être appliqué à d'autres établissements ayant choisi une organisation similaire du pool d'IADE. Dans cet hôpital, les infirmiers travaillant à temps complet ou à temps partiel sont tous poly-compétents et peuvent être affectés indifféremment à l'une des vacations suivantes :

\begin{tabular}{ll} 
Type de vacation & Horaire \\
\hline Journée anesthésie réglée (JR) & $8 \mathrm{~h}-16 \mathrm{~h}$ \\
\hline
\end{tabular}




\begin{tabular}{ll}
\hline Journée anesthésie d'urgence (JU) & $8 \mathrm{~h}-20 \mathrm{~h}$ \\
Nuit anesthésie d'urgence (NU) & $20 \mathrm{~h}-8 \mathrm{~h}$ \\
Supervision SSPI (SS) & $9 \mathrm{~h}-17 \mathrm{~h}$ ou $11 \mathrm{~h}-19 \mathrm{~h}$ \\
\hline
\end{tabular}

Tableau 1. Types de vacations - heure de début et de fin

Les vacations d'urgences (JU et NU) doivent être assurées tous les jours de la semaine (samedi et dimanche compris), alors que les autres vacations ne concernent que les jours allant du lundi au vendredi. La supervision de la SSPI est assurée en décalé par deux IADE avec des horaires différents (afin de couvrir une plus grande durée de supervision de la SSPI). Par la suite, cette différence d'horaires n'engendrant pas de contraintes supplémentaires, nous ne différencions pas les horaires décalés et nous considérons un seul poste qui nécessite deux personnes pendant 8 heures. La surveillance de la SSPI est assurée la nuit en semaine et le week-end 24h sur 24 par des IDE (Infirmier Diplômé d'Etat) postés en réveil, dont la planification constitue un problème indépendant. Ce type d'organisation du travail se retrouve dans la plupart des blocs opératoires, assurant une part d'activité programmée et une part d'activité d'urgences. Cependant, certains établissements ont fait le choix de mettre en place un système d'astreintes à domicile se faisant sur la base du volontariat plutôt qu'un système de garde sur place : un forfait de base est versé pour chaque astreinte tenue, ainsi qu'un supplément pour les heures passées dans l'enceinte de l'hôpital, temps de travail qui n'est pas décompté du temps total. L'affectation des astreintes est donc un problème indépendant de celui d'affectation des activités de jour.

Mode de planification. Le planning des infirmiers anesthésistes est construit par le cadre de santé en suivant un processus non cyclique. En premier lieu, le cadre de santé collecte les desiderata des infirmiers concernant les jours de congés, puis il élabore le planning qui satisfait toutes les contraintes (listées ci-dessous) tout en prenant en compte les préférences personnelles. Etant donné que les infirmiers sont sujets à un temps de travail annualisé, le 
surplus de temps travaillé au cours d'une semaine sera rééquilibré sur les semaines qui suivent par une charge inférieure.

Dans cette organisation un processus de planification non cyclique a été préféré à une planification cyclique, qui se trouve être plus rigide et moins flexible face aux changements. En règle générale, dans tout bloc opératoire, les besoins varient peu d'une semaine à l'autre (hormis pendant les périodes de vacances scolaires) car le programme opératoire journalier prévoit l'ouverture d'un nombre relativement constant de salles, et les vacations d'urgences doivent être assurées chaque jour. Cependant, il arrive que des salles ne soient finalement pas ouvertes (plage opératoire non remplie, chirurgien en formation, etc.), ce qui modifie la table des besoins, et rend la planification non cyclique plus adaptée. Ce type de planification est également plus flexible en ce qui concerne la prise en compte des préférences des personnels au niveau des jours de congés. Celles-ci sont considérées a priori et sont respectées dans la mesure du possible, sachant que l'effectif fixé correspond à l'équipe en place et n'inclut pas le recours au personnel supplémentaire.

Tous les blocs opératoires n'adoptent pas nécessairement le principe des plannings non cycliques et le non cloisonnement en équipes jour/nuit: ce choix dépend fortement des préférences des infirmiers, mais également des dispositifs internes à l'hôpital mis en place pour faciliter la coordination vie personnelle/vie professionnelle des employés (crèche, flexibilité du travail, etc.).

Contraintes. Pour l'élaboration d'un planning, certaines règles sont à respecter. Elles sont issues de la réglementation mais également des accords convenus entre responsables et employés. Les C1 à C4 sont des contraintes « invariantes » que l'on retrouve dans tout type de problème de génération de planning : 
C1 : Les besoins journaliers en nombre d'IADE pour chaque poste doivent être respectés afin de correspondre à la charge de travail.

C2 : Le temps de travail par jour ne doit pas excéder 12 heures par jour.

C3 : Le temps de travail par semaine travaillée doit s'approcher de 38h. Il peut le dépasser dans la limite de $48 \mathrm{~h}$ par semaine (ces seuils peuvent varier d'une convention à l'autre).

C4 : Par semaine, un employé ne doit pas travailler plus de trois nuits. Cette contrainte provient de la limitation du travail de nuit à 40 heures par semaine.

Les contraintes C5 à C6 sont spécifiques aux règles de planification suivies par le cadre de santé en charge de la planification des IADE dans l'hôpital qui constitue notre terrain d'expérience. Elles englobent une part de contraintes réglementaires (repos journalier) mais sont principalement issues de «bonnes pratiques » qui permettent d'obtenir des plannings donnant satisfaction au personnel en terme de confort de travail ainsi qu'à l'encadrement. Ces règles sont cependant facilement modifiables pour s'adapter à d'autres procédures l'élaboration de planning :

C5 : Travailler le samedi de jour JU (respectivement de nuit NU) implique que le dimanche est travaillé de jour JU (respectivement de nuit NU) et que le lundi et le mardi suivants sont des repos.

C6 : Des contraintes de succession d'activité qui permettent entre autres de garantir un repos journalier minimum de 11 heures (figure 2), sont à respecter.

○ Si un infirmier est affecté à une nuit d'urgence NU, alors le lendemain est un repos.

- Si un infirmier est affecté à une journée d'urgence JU, alors le lendemain est soit une nuit d'urgence, soit un repos. Les besoins pour 
les vacations JU étant deux fois plus grands que pour les vacations NU, la moitié des infirmiers assurant une JU un jour donné seront affectée à une NU le lendemain, les autres seront au repos.

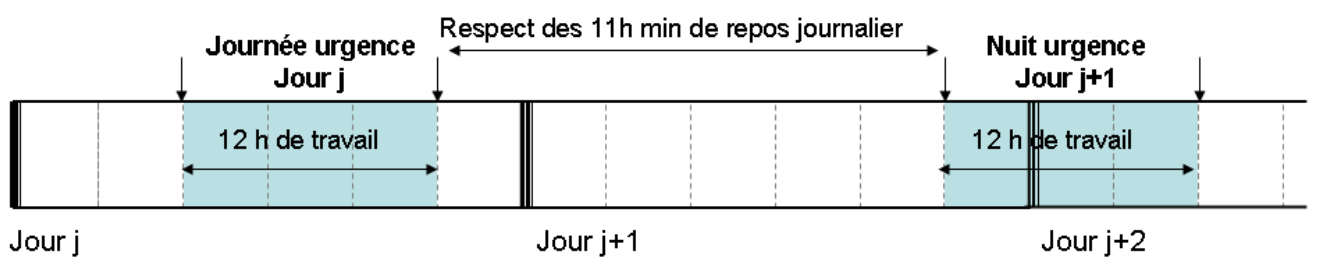

Figure 2. Illustration de la contrainte C6

Les contraintes $\mathrm{C} 1, \mathrm{C} 2, \mathrm{C} 3$ et $\mathrm{C} 4$ sont des contraintes obligatoires que doit respecter tout planning (hard constraints). Les contraintes C5 et C6 expriment des nécessités de repos journalier et de repos hebdomadaire ainsi que des règles issues de «bonnes pratiques » (travail le week-end complet, repos après une nuit). Ces contraintes qui ne relèvent pas de textes réglementaires sont souvent considérées dans la littérature comme des contraintes souples, dites soft contraints, que l'on s'autorise à ne pas satisfaire, mais dont on va chercher le respect «autant que possible». Notre objectif étant d'aboutir à un planning maximisant la satisfaction du personnel, nous cherchons une solution satisfaisant l'ensemble des contraintes (de C1 à C6). Cependant, lorsqu'un problème est très contraint, on se heurte à la difficulté de trouver un planning faisable respectant l'ensemble des contraintes. Dans ce cas, les contraintes souples qui traduisent le plus souvent le confort de travail des employés (positionnement des repos, travail de week-end) sont introduites dans la fonction objectif à optimiser : l'objectif sera de minimiser le nombre de fois où les contraintes souples sont transgressées dans le planning.

Critère à optimiser. Lors de la construction d'un planning, le but est de trouver le meilleur compromis entre trois critères : le coût, la qualité de service et la satisfaction sociale (figure 1). Selon les situations certains critères sont privilégiés par rapport aux autres. 
Dans le problème de planification des infirmiers anesthésistes qui nous intéresse, où l'effectif est considéré constant et complet, le critère de coût n'intervient pas. Il serait cependant à prendre en compte si l'effectif était insuffisant et devait être complété par le travail de personnel supplémentaire. Il faudrait alors identifier le coût des personnels supplémentaires correspondant à l'écart entre le niveau de couverture requis et le personnel disponible comme dans (Jaumard et al., 1998).

La sous-capacité n'est pas autorisée dans la résolution de ce problème. Le critère de qualité de service, qui est évalué par le respect des contraintes $\mathrm{C} 1$ (couverture de la charge) qui sont des contraintes obligatoires, n'intervient donc pas dans la fonction à optimiser.

C'est l'optimisation du critère de satisfaction sociale qui est recherché dans le problème rencontré et qui entre en jeu dans notre fonction objectif. Les contraintes C5 et C6, qui expriment en partie des contraintes de confort de travail, pourraient entrer dans une composante de cette fonction objectif. Nous avons fait le choix de rechercher des plannings qui satisfont tous ces aspects que nous introduisons dans les contraintes du modèle et non dans l'objectif. L'objectif est de générer un planning des infirmiers anesthésistes qui respecte les contraintes $\mathrm{C} 1$ à $\mathrm{C} 6$ tout en maximisant l'équité de la répartition de la charge travail dans l'affectation aux différents postes. En effet, il est primordial de faire tourner régulièrement le personnel sur différentes activités afin d'équilibrer la charge de travail entre les agents, mais aussi dans le but de maintenir les compétences de chaque infirmier dans les différentes spécialités.

Afin de produire le planning le plus équitable possible entre les infirmiers, les vacations les plus populaires et les vacations les moins populaires doivent être réparties d'une manière équilibrée, en prenant en compte la difficulté liée à la réalisation de chaque activité. Afin de traduire la popularité des vacations, nous avons associé une pénibilité à chaque type de 
vacation et à chaque jour de la semaine. Ces pénalités sont comprises dans l'intervalle $[1,2]$ (tableau 2). Les valeurs des pénibilités de chaque poste ont été élaborées en traduisant les connaissances du cadre de santé d'anesthésiologie. Selon lui, on peut classer les pénibilités $\mathrm{P}$ des vacations de la moins pénible à la plus pénible de la manière suivante :

\section{$P($ Journée Réglée $)<P($ Journée Urgence $)<P($ Nuit Urgence $)<P($ Surveillance SSPI $)$}

Il peut paraître surprenant que le poste de Surveillance de SSPI soit plus pénible à occuper que celui de Nuit Urgence. Ceci est du au fait que l'activité de SSPI, assuré par un personnel limité pour quatorze places, est beaucoup plus morcelée dans le temps et demande une grande vigilance à cette phase souvent critique de réveil. Les postes d'urgence, de journée ou de nuit, bien qu'étant des horaires de 12 heures sont finalement plus appréciés des infirmiers. Enfin, la vacation de Journée Réglée, correspondant à l'activité de surveillance de l'anesthésie en salle d'intervention et étant la plus commode au niveau des horaires et de l'activité, elle possède la pénibilité la plus faible.

\begin{tabular}{lccccccc} 
Type de vacation & Lun & Mar & Mer & Jeu & Ven & Sam & Dim \\
\hline Journée Réglé (JR) & 1 & 1 & 1 & 1 & 1 & 0 & 0 \\
Journée Urgence (JU) & 1.2 & 1.2 & 1.2 & 1.2 & 1.2 & 1.4 & 1.4 \\
Nuit Urgence (NU) & 1.4 & 1.4 & 1.4 & 1.4 & 1.4 & 1.6 & 1.6 \\
Surveillance SSPI (SS) & 1.6 & 1.6 & 1.6 & 1.6 & 1.6 & 0 & 0 \\
\hline
\end{tabular}

Tableau 2. Pénibilités associées aux types de vacations

Le meilleur planning sera celui qui minimise la pénibilité totale associée à chaque employé. Afin de trouver le planning assurant la plus petite pénibilité totale pour chaque infirmier, notre objectif sera de minimiser la différence entre l'infirmier le plus chargé et l'infirmier le moins chargé. D'autres critères, comme la minimisation de la pénibilité totale de l'employé le plus chargé ou encore la maximisation de la pénibilité totale de l'employé le moins chargé ont été envisagés. Apres une série d'expérimentation, notre choix s'est porté sur la minimisation de 
l'écart entre les pénibilité des infirmiers, qui donne le plus satisfaction quant à la réduction de la disparité dans la distribution des tâches.

Ces notions de pénibilité sont à manipuler avec précaution. Elles sont très fortement corrélées au système dans lequel on se place. Grandeur difficile à quantifier, elles dépendent de la perception des personnes, des habitudes de travail, des contraintes personnelles. Dans les expérimentations de la section 4 , nous testons un second jeu de pénibilités, afin de tester la sensibilité de notre modèle.

Le problème spécifique que nous avons exposé comprend une part de «standard » et une part de «spécifique» qui peut être adapté à d'autres habitudes de travail et à d'autres organisations. La modélisation du problème à l'aide de la programmation mathématique que nous exposons dans la section suivante (3.2) permet précisément d'adapter le modèle à des évolutions dans le temps voire à d'autres réalités de manière aisée.

Dans (Trilling et al., 2006), nous avons comparé plusieurs techniques : la programmation linéaire en nombres mixtes (PL) et la programmation par contraintes (PPC) pour la résolution de ce problème d'affectation des infirmiers anesthésistes. En termes de modélisation, le fort pouvoir d'expressivité de la PPC permet d'une part de limiter le nombre de variables manipulées mais aussi de faciliter l'expression de certaines contraintes globales. Au niveau de l'optimisation, la procédure de séparation et évaluation des solveurs de PL est plutôt efficace, bien qu'opaque. En PPC, la valeur de l'objectif de la première solution trouvée a un fort impact sur la vitesse de convergence vers l'optimum. D'où l'importance de développer une stratégie de recherche pour cette approche permettant d'obtenir une bonne solution dès la première itération. A la lumière des résultats de ces travaux, nous avons pu pouvons conclure, que dans l'état actuel de perfectionnement de chacune des approches, l'approche PL montrait sa supériorité pour ce problème spécifique, en termes de valeur de la fonction objectif obtenu 
et de temps de réponse, ainsi qu'une certaine facilité de mise en oeuvre. Elle permet d'aboutir plus rapidement à un planning des infirmiers dans lequel la charge est relativement bien équilibrée entre les individus. Ceci nous a amené à privilégier l'utilisation de la programmation linéaire dans la suite de nos travaux. La modélisation de ce problème d'affectation sous la forme d'un programme linéaire à variables mixtes est présentée dans la section suivante.

\subsection{Modélisation du problème}

Variables. Dans la formulation du PPIA sous forme d'un programme linéaire à variables mixtes, les principales variables de décision sont les variables binaires $X_{i j k}$ qui correspondent à l'affectation de l'infirmier $i(1 \leq i \leq N)$, le jour $j(1 \leq j \leq H)$, à la vacation $k(1 \leq k \leq S)$ et prennent la valeur 0 ou 1 :

$$
X_{i j k}= \begin{cases}1 & \text { l'infirmier } i \text { travaille durant la vacation } k \text { le jour } j \\ 0 & \text { sinon. }\end{cases}
$$

D'autres variables, continues cette fois-ci, sont nécessaires pour la modélisation de ce problème. Il s'agit de la pénibilité de l'infirmier le plus chargé $P_{\max }$ et celle de l'infirmier le moins chargé $P_{\min }$. Ces deux variables seront utilisées dans la fonction objectif.

Objectif. Le but est ici de générer un planning dans lequel la charge est distribuée de manière équilibrée entre les infirmiers, en tenant compte de la difficulté (concentration, fatigue physique) liée à chaque tâche. Nous proposons de traduire cet objectif d'équité en utilisant des pénibilités $P_{j k}$, pour chaque jour $j$ et chaque vacation $k$. Les valeurs de $P_{j k}$ sont données dans le tableau 2. Ainsi la fonction objectif peut être formulée de la manière suivante : 


$$
\text { Minimiser } \quad Z=P_{\max }-P_{\text {min }}
$$

où $P_{\max }$ est la borne supérieure de la pénibilité totale de chaque infirmier (correspondant à la pénibilité totale de l'infirmier le plus chargé) et $P_{\min }$ la borne inférieure. Ces bornes sont définies à l'aide des contraintes suivantes, dans lesquelles $R_{i}$ désigne le régime de travail de chaque infirmier ( $=1$ pour un temps complet, $<1$ pour un temps partiel) :

$$
\begin{aligned}
& P_{\min }-\left(\sum_{j=1}^{H} \sum_{k=1}^{S} \frac{1}{R_{i}} \cdot X_{i j k} \cdot P_{j k}\right) \leq 0 \quad \forall i=1, \ldots N \\
& P_{\max }-\left(\sum_{j=1}^{H} \sum_{k=1}^{S} \frac{1}{R_{i}} \cdot X_{i j k} \cdot P_{j k}\right) \geq 0 \quad \forall i=1, \ldots N
\end{aligned}
$$

Contraintes. Il n'est pas aisé de formuler certaines contraintes en programmation mathématique, en particulier celles concernant la succession d'activité (JU suivi soit d'une NU soit d'un repos). Pour cette raison nous avons introduit un nouveau type de vacation JU', ce qui amène à considérer non plus 4 mais 5 vacations possibles : JR $(k=1)$, JU $(k=2)$, JU' $(k=3), \mathrm{NU}(k=4)$ et SS $(k=5)$.

Les contraintes de couverture $\mathbf{C 1}$ sont exprimées par l'équation (4), où $B_{j k}$ représente le nombre d'infirmiers requis pour la vacation $k$ le jour $j$ :

$$
\sum_{i=1}^{N} X_{i j k}=B_{j k} \quad \forall k=1, \ldots, S \quad \forall j=1, \ldots, H
$$

Les contraintes C2 limitant le nombre d'heures travaillées par jour sont traduites par l'équation (5) qui n'autorise l'affectation que d'une seule vacation par jour et par infirmier :

$$
\sum_{k=1}^{S} X_{i j k} \leq 1 \quad \forall i=1, \ldots, N \quad \forall j=1, \ldots, H
$$


Les contraintes C3, concernant la limite des heures travaillées par semaine sont formulées en prenant en compte le régime de travail $R_{i}$ de chaque employé, ainsi que $N_{k}$ le nombre d'heures travaillées durant la vacation $k$ :

$$
\sum_{j=1}^{H} \sum_{k=1}^{S} X_{i j k} \cdot N_{k} \leq \frac{H}{7} \cdot 48 \cdot R_{i} \quad \forall i=1, \ldots, N
$$

Les contraintes $\mathbf{C 4}$, limitant le nombre de nuits par semaine, sont exprimées à l'aide d'un nouvel indice $h$ correspondant à la semaine $(1 \leq h \leq H / 7)$.

$$
\sum_{j=7 h-6}^{7 h} X_{i j 4} \leq 3 \quad \forall i=1, \ldots, N \quad \forall h=1, \ldots, \frac{H}{7}
$$

Les contraintes $\mathbf{C 5}$, concernant le travail du week-end nécessite plusieurs équations:

- La même vacation est affectée aux deux jours du week-end, ou bien le week-end est au repos :

$$
X_{i,(7 h-1), k}-X_{i, 7 h, k}=0 \quad \forall i=1, \ldots, N \quad \forall h=1, \ldots, \frac{H}{7} \quad \forall k=3,4
$$

- Après un week-end travaillé les deux jours suivants sont au repos :

$$
X_{i, 7 h, k}+\sum_{k^{\prime}=1}^{S} X_{i, 7 h+d, k^{\prime}} \leq 1 \quad \forall d=1,2 \quad \forall i=1, \ldots, N \quad \forall h=1, \ldots, \frac{H}{7}-1 \quad \forall k=3,4
$$

Les contraintes de succession d'activités C6 concernent uniquement les jours de la semaine (hors week-end) :

- Chaque JU et chaque NU doivent être suivis par un repos:

$$
X_{i, 7 h-7+j, k}+\sum_{k^{\prime}=1}^{S} X_{i, 7 h-6+j, k^{\prime}} \leq 1 \quad \forall k=2,4 \quad \forall i=1, \ldots, N \quad \forall j=1, \ldots, 5 \quad \forall h=1, \ldots, \frac{H}{7}
$$


- Chaque JU' doit être suivi par un NU:

$$
X_{i, 7(h-1)+j, 3}-X_{i, 7(h-1)+j+1,4}=0 \quad \forall i=1, \ldots, N \forall j=1, \ldots, 4 \forall h=1, \ldots, \frac{H}{7}
$$

Finalement, l'intégration des préférences des infirmiers est rendue possible par l'ajout de contraintes forçant les variables $X_{i j k}$ à prendre la valeur 0, lorsque l'infirmier $i$ souhaite disposer d'un congé le jour $j$ (pour toutes les activités $k$ ):

$$
X_{i j k}=0 \quad \forall k=1, \ldots, 5
$$

Résolution. Ce modèle a été validé en premier lieu à l'aide du solveur commercial LINGO 8.0 (LINDO, 2003), en résolvant le problème tel qu'il a été rencontré à l'hôpital (besoin, effectifs, pénalités). Par la suite, il nous a semblé intéressant de tester d'autres solveurs afin d'éprouver les résultats trouvés par LINGO. La description de la démarche, les jeux de tests et les résultats sont explicités dans la section suivante.

\section{Comparaison des résultats sur différents solveurs}

Le solveur LINGO dont nous avons disposé pour la validation du modèle n'est pas connu comme étant le meilleur solveur du marché (Neumaier et al., 2005), les performances dépendant bien évidement des problèmes résolus. Il est néanmoins plus accessible financièrement que d'autres solveurs commerciaux comme CPLEX ${ }^{1}$, ce qui explique notre orientation première. Il nous a tout de même semblé judicieux de tester le modèle sur un solveur de référence, CPLEX (ILOG, 2001), réputé pour ses performances en termes de temps

\footnotetext{
${ }^{1}$ La licence éducation de LINGO est accessible à partir de 700 US\$ pour des problèmes atteignant 3200 variables entières (1200 US\$ pour la version illimitée) tandis que la licence éducation de CPLEX s'élève à $1400 €$.
} 
de calcul. Parallèlement à cela, dans le but de rendre notre modèle directement utilisable par les cadres de santé, sans leur imposer d'acquisition de logiciels payants que sont les solveurs commerciaux, nous avons implémenté le modèle à l'aide du solveur libre GLPK (GLPK, 2005). Ceci nous permettant de valider ou non la faisabilité d'utiliser un logiciel libre pour la résolution d'un tel problème. Afin d'éprouver la qualité du modèle et la capacité des solveurs à le résoudre, nous avons réalisé une série de tests correspondant à des tailles de problèmes différents. Plusieurs paramètres font l'objet de variations :

- L'horizon temporel $\mathrm{H}$ : il s'agit du nombre de jours considéré dans le planning ;

- La taille de l'effectif des personnels $\mathrm{N}$ : ce paramètre est directement relié à d'autres paramètres d'entrées qui sont :

○ les besoins $B_{j k}$ en nombre de personnes pour chaque jour et chaque vacation,

○ le régime de travail $R_{i}$ de chaque infirmier de l'effectif (temps complet, $80 \%, 70 \%, 50 \%$ ou $30 \%^{2}$ )

○ La valeur de la pénibilité $P_{j k}$ associée à chaque vacation et à chaque jour de la semaine.

\begin{tabular}{|ccccccccc|}
\hline $\begin{array}{c}\text { Pénibilité } \\
\text { 1 }\end{array}$ & Lun & Mar & Mer & Jeu & Ven & Sam & Dim \\
\cline { 1 - 7 } JR & 1 & 1 & 1 & 1 & 1 & 0 & 0 \\
JU & 1,2 & 1,2 & 1,2 & 1,2 & 1,2 & 1,4 & 1,4 \\
NU & 1,4 & 1,4 & 1,4 & 1,4 & 1,4 & 1,6 & 1,6 & \\
SS & 1,6 & 1,6 & 1,6 & 1,6 & 1,6 & 0 & 0 \\
\hline Besoin 1 & Lun & Mar & Mer & Jeu & Ven & Sam & Dim & Effectif \\
\hline JR & 2 & 2 & 2 & 2 & 2 & 0 & 0 & \\
JU & 2 & 2 & 2 & 2 & 2 & 1 & 1 & \multirow{1}{*}{$\mathbf{1 4}$} \\
NU & 1 & 1 & 1 & 1 & 1 & 1 & 1 & \\
SS & 2 & 2 & 2 & 2 & 2 & 0 & 0 & \\
\hline
\end{tabular}

\begin{tabular}{|ccccccccc|}
\hline $\begin{array}{c}\text { Pénibilité } \\
\text { 2 }\end{array}$ & Lun & Mar & Mer & Jeu & Ven & Sam & Dim \\
\hline JR & 1 & 1 & 1 & 1 & 1 & 0 & 0 \\
JU & 1,4 & 1,4 & 1,4 & 1,4 & 1,4 & 1,6 & 1,6 & \\
NU & 1,4 & 1,4 & 1,4 & 1,4 & 1,4 & 1,6 & 1,6 \\
SS & 1,2 & 1,2 & 1,2 & 1,2 & 1,2 & 0 & 0 & \\
\hline \hline Besoin 2 & Lun & Mar & Mer & Jeu & Ven & Sam & Dim & Effectif \\
\hline JR & 7 & 7 & 7 & 7 & 7 & 0 & 0 & \\
JU & 2 & 2 & 2 & 2 & 2 & 1 & 1 & \multirow{2}{22}{} \\
NU & 1 & 1 & 1 & 1 & 1 & 1 & 1 & \\
SS & 2 & 2 & 2 & 2 & 2 & 0 & 0 & \\
\hline
\end{tabular}

\footnotetext{
${ }^{2}$ Les personnes dont le régime de travail est à 30\% interviennent dans plusieurs services et ne passent que $30 \%$ de leur temps au bloc opératoire.
} 


\begin{tabular}{|ccccccccc|}
\hline Besoin 3 & Lun & Mar & Mer & Jeu & Ven & Sam & Dim & Effectif \\
\hline JR & 10 & 10 & 10 & 10 & 10 & 0 & 0 & \\
JU & 2 & 2 & 2 & 2 & 2 & 1 & 1 & $\mathbf{2 6}$ \\
NU & 1 & 1 & 1 & 1 & 1 & 1 & 1 & \\
SS & 3 & 3 & 3 & 3 & 3 & 0 & 0 & \\
\hline
\end{tabular}

\begin{tabular}{|ccccccccc|}
\hline Besoin 4 & Lun & Mar & Mer & Jeu & Ven & Sam & Dim & Effectif \\
\hline JR & 20 & 20 & 20 & 20 & 20 & 0 & 0 & \\
JU & 4 & 4 & 4 & 4 & 4 & 2 & 2 & 52 \\
NU & 2 & 2 & 2 & 2 & 2 & 2 & 2 & \\
SS & 4 & 4 & 4 & 4 & 4 & 0 & 0 & \\
\hline
\end{tabular}

\section{Tableau 3. Jeux d'essais expérimentés sur 7, 14 et 28 jours à l'aide de CPLEX, LINGO} et GLPK

Les différentes valeurs des paramètres qui ont été testées sont présentées dans le tableau 3.

Nous avons choisi pour chaque besoin une composition d'effectif mixant des personnels travaillant à temps complets et à temps partiels dans des proportions habituellement rencontrées. Les besoins considérés couvrent un ensemble de cas envisageables, allant de l'ouverture de 4 salles (petit bloc de spécialité) à 24 salles (plateau médico-technique regroupé). Par exemple pour le besoin 3, le bloc est composé de 10 salles d'intervention accueillant les patients programmés et de 2 salles dédiées aux urgences, dont une seule est ouverte la nuit. Pour chacune des salles ouvertes, la présence d'un IADE est exigée. Le nombre de poste de réveil en salle de surveillance post-interventionnelle (SSPI) nécessite la présence de 3 IADE chargées de la supervision de la salle dans la journée. Le premier jeu de pénibilités testé a été présenté dans la description du problème. Le second a été construit en prenant en compte, outre la difficulté des vacations, la durée de chacune d'elles (la pénibilité des vacations de journée et nuit d'urgence, d'une durée de 12 heures, ont une pénibilité supérieure à celles de la vacation de supervision de la SSPI). Le planning a été généré pour des horizons de 7, 14 et 28 jours.

Les expérimentations ont été effectuées sur un Pentium M (1500MHz, 504Mo RAM), en choisissant les options des solveurs proposées par défaut. Dans chacun des cas, l'optimum n'a pas été trouvé, mais la plupart du temps la solution obtenue après quelques minutes de recherche est identique à la solution en cours après une heure de recherche. La solution optimale $(Z=0)$ recherchée par les solveurs n'est pas atteignable en un temps raisonnable ; elle correspond néanmoins à une situation idéale difficilement atteignable. Les résultats 
donnés par le tableau 4 sont ceux obtenus après 10 minutes de calcul pour CPLEX et LINGO et après 20 minutes pour GLPK. Le tableau fait apparaître la valeur de la meilleure fonction objectif ( $Z$ ) obtenue pour chacune des expérimentations (à titre d'indication, pour le solveur CPLEX, les solutions retenues et consignées dans le tableau ont été atteintes en un temps de calcul allant de 17 secondes à 9 minutes, avec une moyenne de 3 minutes) ainsi qu'un indicateur d'écart maximum du temps travaillé par les infirmiers tenant compte du régime de travail ( $\Delta T)$. La troisième colonne donne le nombre de variables d'affectation binaires (0-1) du problème considéré.

\section{\begin{tabular}{|l|l|l|}
\hline Horizon & Effectif & $0-1$ \\
\hline
\end{tabular}}

\begin{tabular}{|ccc|}
\hline 7 jours & $\mathrm{N}=14$ & 490 \\
& $\mathrm{~N}=22$ & 770 \\
& $\mathrm{~N}=26$ & 910 \\
& $\mathrm{~N}=52$ & 1820 \\
\hline 14 jours & $\mathrm{N}=14$ & 980 \\
& $\mathrm{~N}=22$ & 1540 \\
& $\mathrm{~N}=26$ & 1820 \\
& $\mathrm{~N}=52$ & 3640 \\
\hline 28 jours & $\mathrm{N}=14$ & 1960 \\
& $\mathrm{~N}=22$ & 3080 \\
& $\mathrm{~N}=26$ & 3640 \\
& $\mathrm{~N}=52$ & 7280 \\
\hline
\end{tabular}

\begin{tabular}{|c|c|c|c|c|c|}
\hline \multicolumn{5}{|c|}{ Pénibilité 1 } \\
\hline \multicolumn{2}{|c|}{ CPLEX } & \multicolumn{2}{|c|}{ LINGO } & \multicolumn{2}{c|}{ GLPK } \\
\hline$Z$ & $\Delta T$ & $Z$ & $\Delta T$ & $Z$ & $\Delta T$ \\
\hline
\end{tabular}

\begin{tabular}{llllll|}
0,60 & 0,57 & 0,80 & 0,68 & 2,40 & 0,84
\end{tabular}

$\begin{array}{llllll}0,47 & 0,31 & 1,20 & 0,70 & 2,50 & 0,60\end{array}$

$\begin{array}{llllll}0,51 & 0,41 & 1,05 & 0,62 & 2,55 & 0,66\end{array}$

\begin{tabular}{llllll|}
0,60 & 0,28 & 1,20 & 0,41 & 3,14 & 0,66 \\
\hline 0,70 & 0,43 & 0,45 & 0,27 & 1,75 & 0,39
\end{tabular}

\begin{tabular}{llllll|}
0,70 & 0,43 & 0,45 & 0,27 & 1,75 & 0,39
\end{tabular}

$\begin{array}{llllll}0,60 & 0,56 & 1,00 & 0,44 & 3,00 & 0,48\end{array}$

$\begin{array}{llllll}0,50 & 0,33 & 1,00 & 0,48 & 2,00 & 0,49\end{array}$

$\begin{array}{llll}1,60 & 0,41 & 2,28 & 0,47\end{array}$

\begin{tabular}{llll}
1,60 & 0,41 & 2,28 & 0,47 \\
\hline 0,40 & 0,15 & 0,75 & 0,74
\end{tabular}

$\begin{array}{llll}0,97 & 0,35 & 0,14 & 0,28\end{array}$

$\begin{array}{llll}0,86 & 0,27 & 1,20 & 0,28\end{array}$

$1,09 \quad 0,38$

$6,60 \quad 0,84$

\begin{tabular}{|c|c|c|c|c|c|}
\hline \multicolumn{6}{|c|}{ Pénibilité 2} \\
\hline \multicolumn{2}{|c|}{ CPLEX } & \multicolumn{2}{|c|}{ LINGO } & \multicolumn{2}{|c|}{ GLPK } \\
\hline$Z$ & $\Delta T$ & $Z$ & $\Delta T$ & $Z$ & $\Delta T$ \\
\hline 0,75 & 0,28 & 0,80 & 0,68 & 1,75 & 0,30 \\
\hline 0,75 & 0,28 & 1,20 & 0,70 & 2,42 & 0,50 \\
\hline 0,80 & 0,28 & 1,05 & 0,62 & 1,75 & 0,30 \\
\hline 0,86 & 0,29 & 1,20 & 0,77 & 3,75 & 0,80 \\
\hline 0,75 & 0,15 & 0,85 & 0,15 & 2,00 & 0,28 \\
\hline 0,6 & 0,15 & 1,20 & 0,22 & 2,40 & 0,47 \\
\hline 1,20 & 0,21 & 0,95 & 0,44 & 3,47 & 0,43 \\
\hline 0,85 & 0,14 & 2,00 & 0,28 & 6,33 & 0,60 \\
\hline 0,20 & 0,09 & 1,05 & 0,11 & 2,25 & 0,18 \\
\hline 0,75 & 0,09 & 1,20 & 0,17 & 4,47 & 0,37 \\
\hline 0,95 & 0,14 & 1,25 & 0,16 & 3,40 & 0,21 \\
\hline 1,00 & 0,14 & 2,2 & 0,24 & & \\
\hline
\end{tabular}

Tableau 4. Résultats : 24 expérimentations sur 3 solveurs.

La figure 3 présente un exemple de résultat obtenu par LINGO pour l'un des problèmes (22 infirmiers, sur 14 jours). Ce tableau élaboré automatiquement à partir des résultats donnés par le solveur, nous permet de vérifier visuellement la répartition relativement homogène des vacations parmi les infirmiers (en prenant en considération leur régime de travail).

On remarque que le solveur CPLEX, pour la plupart des expérimentations, trouve un objectif inférieur à 1 , ce qui est un bon résultat par rapport à un planning réalisé manuellement. La courbe de recherche de ce solveur atteint une valeur asymptotique au bout de quelques secondes (ou minutes selon les expérimentations) qui ne s'améliore pas en prolongeant la 
recherche. En général plus le problème est petit, plus l'objectif est bon, cependant cette évolution, au vu du tableau de résultats, n'est pas totalement linéaire. Avec le second jeu de pénibilités, on obtient des valeurs de l'objectif légèrement supérieures, avec toutefois, un écart du temps travaillé moins élevé qu'avec le premier jeu de pénibilités.

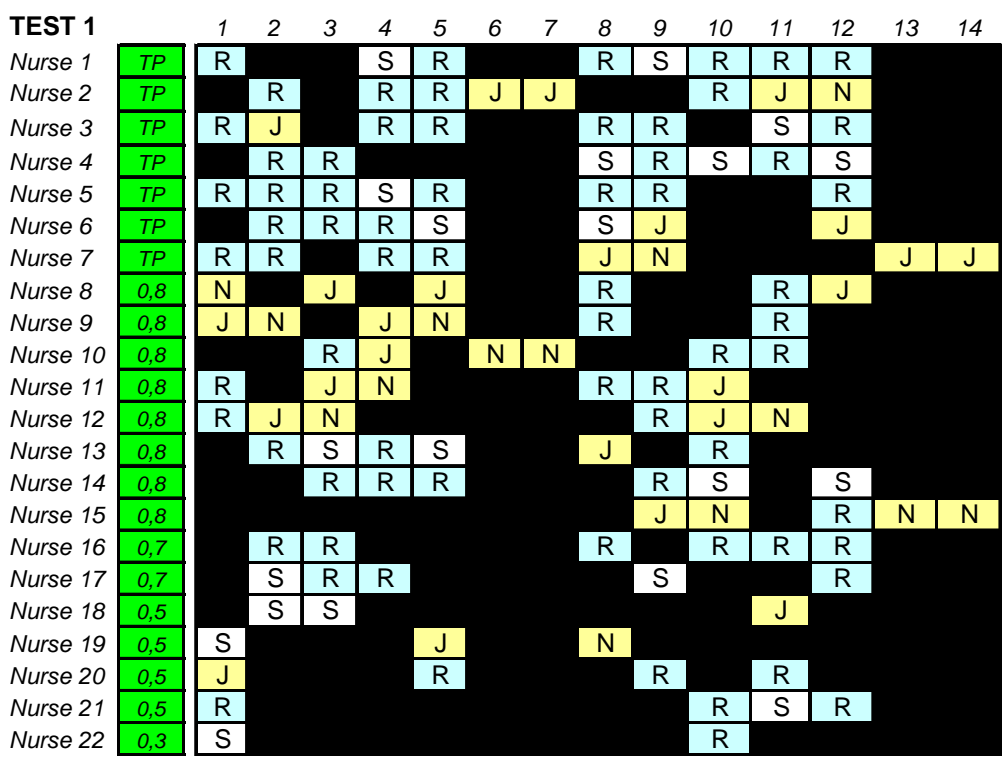

Légende :

$\mathrm{R}=$ Journée Réglée

$\mathrm{J}=$ Journée Urgence

$\mathrm{N}=$ Nuit Urgence

$\mathrm{S}=$ Surveillance SSPI

$\mathrm{TP}=$ Temps plein

Figure 3. Exemple de planning obtenu par LINGO

La valeur de l'objectif donnée par le solveur LINGO est presque toujours légèrement supérieure à celui donné par CPLEX. Les exceptions peuvent s'expliquer par le fait que chaque solveur utilise des algorithmes différents, avec une procédure de séparation et évaluation qui leurs est propre, et qui conduisent les recherches vers des solutions différentes. L'écart d'objectif trouvé s'accentue pour des problèmes de très grande taille (avec le plus grand effectif et pour un horizon supérieur à 14 jours).

Les performances du solveur libre GLPK sont assez décevantes en comparaison de celles des autres solveurs. Même avec un temps de recherche deux fois supérieur, les résultats trouvés sont nettement moins bons. Pour le plus grand problème (52 infirmiers, horizon de 28 jours), aucune solution faisable n'a pu être déterminée. Sa procédure de séparation et évaluation étant assez basique, ce solveur libre résout avec difficulté les problèmes linéaires mixtes 
comprenant un grand nombre de variables entières tels que le nôtre. Pour les grandes tailles de problème, l'utilisation d'un solveur du marché est donc indispensable à la résolution de ce problème dans l'état actuel de la modélisation.

La qualité des plannings obtenus à l'aide des solveurs commerciaux est aussi bonne, sinon meilleure que ceux réalisés manuellement par le cadre de santé, qui peut passer largement une demi-journée par semaine à l'élaboration des plannings alors qu'un solveur trouve une bonne solution en moins de 10 minutes. Les cadres de santé ont montré un grand intérêt à utiliser un solveur pour les aider dans l'élaboration des plannings infirmiers et leur épargner les longues vérifications du respect de l'ensemble des contraintes. Le coût d'investissement pouvant paraître rédhibitoire pour un usage unique limité à un service, deviendrait largement acceptable si l'utilisation d'un tel outil était généralisée à l'ensemble des services de l'hôpital.

\section{Conclusion}

Nous avons présenté un modèle d'affectation des infirmiers anesthésistes, dont l'objectif est la recherche d'une équité maximale entre les infirmiers. Le modèle présenté comporte une partie générique ainsi qu'une partie spécifique à l'organisation étudiée. Il est facilement généralisable à d'autres organisations.

L'utilisation de solveurs pour la résolution du problème de planification des infirmiers présente un intérêt pratique reconnu par les cadres de santé. Ils sont favorables à l'appropriation d'une aide à l'élaboration d'un planning, qui constitue habituellement une tâche fastidieuse occupant une part non négligeable de leur temps. La résolution de problèmes de tailles variées sur différents solveurs a montré la nette supériorité des solveurs commerciaux CPLEX et LINGO face au solveur libre GLPK. Ce constat permet d'émettre une réserve quant à l'utilisation d'un solveur libre pour la résolution de ce type de problème 
au quotidien. Les solveurs commerciaux peuvent constituer une alternative intéressante si leur emploi pour la gestion du personnel est déployé sur plusieurs services (voire même exploité pour d'autres problèmes d'optimisation).

Afin de faciliter la résolution du PPIA par le solveur libre GLPK, il serait utile de scinder le problème en plusieurs sous-problèmes, tels que : (1) l'affectation des vacations d'urgence qui sont soumises à des contraintes fortes (temps de travail, repos) ; (2) l'affectation des vacations de journée réglé et de surveillance de SSPI, qui sont toutes deux des vacations de jour d'une durée de 8 heures, pouvant être facilement inter-changées. Chacun des sous-problèmes comportant un nombre moins élevé de variables, il serait alors plus aisément résolu. Il faudrait cependant articuler la résolution des sous-problèmes et recomposer la solution finale.

\section{Remerciements}

Les auteurs adressent leurs remerciements aux rapporteurs anonymes pour leurs commentaires avisés qui ont permis d'améliorer sensiblement le contenu de ce papier. Ce travail a été réalisé dans le cadre des actions menées pour le projet $\mathrm{HRP}^{2}$ (Hôpitaux: Regroupement, Partage et Pilotage), financé de 2003 à 2006 par la Région Rhône-Alpes sur les thématiques prioritaires de transfert de technologie et d'innovation.

\section{Bibliographie}

Abdennadher, S.,Schlenker, H. (1999). Nurse scheduling using constraint logic programming. Proceedings of Innovative Applications of Artificial Intelligence AAAI/IAAI-99, Orlando, Florida.

Aickelin, U.,Dowsland, K. A. (2004). An indirect Genetic Algorithm for a nurse scheduling problem. Computers and Operations Research, vol 31, pp. 761-778.

Berrada, I., Ferland, J. A.,Michelon, P. (1996). A multi-objective approach to nurse scheduling with both hard and soft constraints. Socio-Economic Planning Sciences, vol 30, pp. 183-193.

Blöchliger, I. (2004). Modeling staff scheduling problem: A tutorial. European Journal of Operational Research, vol 158, pp. 533-542. 
Brusco, M. J.,Jacobs, L. W. (1995). Cost analysis of alternative formulations for personnel scheduling in continuously operating organisations. European Journal of Operational Research, vol 86, pp. 249-261.

Burke, E. K., Causmaecker, P. D., Berghe, G. V.,Landeghem, H. V. (2004). The state of the art of nurse rostering. Journal of scheduling, vol $7 \mathrm{n}^{\circ} 6$, pp. 441-499.

Chan, P. (2002). La planification du personnel: acteurs, actions et termes multiples pour une planification opérationnelle des personnes. Thèse de doctorat, Université Joseph Fourier de Grenoble.

Ernst, A. T., Jiang, H., Krishnamoorthy, M.,Sier, D. (2004). Staff scheduling and rostering : A review of applications, methods and models. European Journal of Operational Research, vol $153 \mathrm{n}^{\circ} 1$, pp. 3-27.

GLPK (2005). GNU Linear Programming Kit : Reference Manual Version 4.8.

GMSIH (2002). Analyse de l'offre du marché concernant les logiciels de gestion des temps. http://www.gmsih.fr/tiki-view_tracker.php?trackerId=1.

ILOG (2001). CPLEX 7.1 User's Manual.

Jaumard, B., Semet, F.,Vovor, T. (1998). A generalised linear programming model for nurse scheduling. European Journal of Operational Research, vol 107, pp. 1-18.

LINDO (2003). LINGO 8.0 User's Manual, Lindo System Inc.

Neumaier, A., Shcherbina, O., Huyer, W.,Vinko, T. (2005). A comparison of complete global optimization solvers. Mathematical Programming, vol 103, pp. 335-356.

Partouche, A. (1998). Planification d'horaires de travail : méthodologies, modélisation et résolution à l'aide de la programmation linéaire en nombres entiers et de la programmation par contraintes. Thèse de doctorat, Université Paris-Dauphine.

SFAR (2002). Commentaires sur le décret ministériel n 2002-194 du 11/02/02 relatif aux actes professionnels et à la profession d'infirmier. http://www.sfar.org/s/.

Tien, J.,Kamiyama, A. (1982). On manpower scheduling algorithms. SIAM Review, vol 24, pp. 275-287.

Trilling, L., Guinet, A.,Le Magny, D. (2006). Nurse scheduling using integer linear programming and constraint programming. Proceedings of INCOM 06, Saint Etienne, France.

Valouxis, C.,Housos, E. (2000). Hybrid optimization techniques for the workshift and rest assignment of nursing personnel. Artificial Intelligence in Medicine, vol 20, pp. 155175.

Weil, G., Heus, K., François, P.,Poujade, M. (1995). Constraint Programming for Nurse Scheduling. IEEE Engineering in Medicine and Biology, vol 14 n$^{\circ} 4$, pp. 417-422. 